\title{
Nonlinear Thrust Controller for Marine Propellers in Four-Quadrant Operations
}

\author{
L. Pivano ${ }^{1,3}$, T. A. Johansen ${ }^{1}, \varnothing$. N. Smogeli ${ }^{2}$ and T. I. Fossen ${ }^{1}$
}

\begin{abstract}
In this paper a nonlinear thrust controller for a marine propeller in four-quadrant operations is presented. It is a shaft speed controller where the desired velocity is computed based on the desired propeller thrust and on the torque losses, estimated with a nonlinear observer. Experimental results are provided to demonstrate the effectiveness of the controller. The proposed scheme shows improved performance in thrust production when compared to traditional shaft speed and torque control.
\end{abstract}

\section{INTRODUCTION}

In marine guidance, navigation and control (GNC) systems, the low level thrusters controller have traditionally received less attention compared to the guidance system and the highlevel control. In the last years also the effect of the thruster and propeller dynamics and control has been studied [6], [12], [2], [7] and [1].

The main difficulties in the design of effective propeller controllers lie in the modeling of the propeller's dynamics and in the problem of measuring the environmental state. A marine propeller is often affected by thrust losses due to in-line velocity variations, ventilation, in-and-out-of water effects, wave-induced water velocities and interaction between the vessel hull and the propeller. A propeller may operate far from the nominal condition and the knowledge of the value of the propeller thrust and torque is thus fundamental to achieve high control performance. Unfortunately, a propeller system is not usually equipped with thrust and torque sensors, therefore thrust losses are not directly measured.

In this paper a nonlinear propeller thrust controller is designed incorporating a thrust loss estimate. The thrust controller consists of a propeller shaft speed controller where the speed reference is computed from the thrust reference given by the high level controller (e.g. a vessel speed controller) and the torque losses, estimated with a nonlinear observer.

Observers for the torque estimation have been presented in [7] and [6] where they were included in anti-spin algorithms in order to detect a ventilation incident, i.e. high loss situation that occurs in extreme seas.

In [8], the classical shaft speed, torque and power control and combination of those have been developed and implemented. All the mentioned controllers do not use any information about the propeller working condition, i.e. the propeller performance may be poor when thrust losses occur. The main contribution of this paper is the estimation of the propeller working condition and the inclusion of this information into

${ }^{1}$ L.Pivano, T. A. Johansen and T.I. Fossen are with Department of Engineering Cybernetics, Norwegian University of Science and Technology, NO-7491 Trondheim, Norway. E-mail: [luca.pivano,tor.arne.johansen,tif]@itk.ntnu.no

${ }^{2} \varnothing$. N. Smogeli is with the R\&D Department, Marine Cybernetics, NO7075 Tiller, Norway [Oyvind.Smogeli@marinecyb.com]

${ }^{3}$ Corresponding author. the controller in order to compensate for the thrust losses. Moreover the proposed controller is designed to work in full four-quandrant plane composed by the vessel and the shaft speed.

\section{PROPELLER MODEL}

Fig. 1 shows the propeller system composed of an electric motor attached to a shaft and to a propeller through a gear-box.

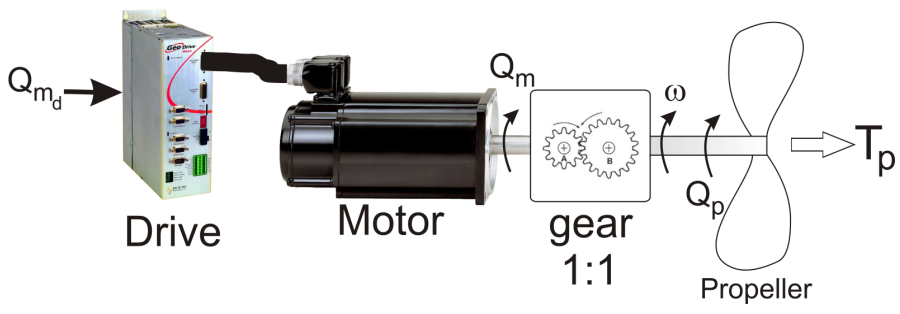

Fig. 1. Propeller system.

The shaft rotates at the angular speed $\omega$. Its dynamics can be written as:

$$
J_{m} \dot{\omega}=Q_{m}-Q_{p}-Q_{f}(\omega),
$$

where $J_{m}$ is shaft moment of inertia, $Q_{f}(\omega)$ is the shaft friction torque which depends on the shaft speed and $Q_{m}$ is the motor torque which is controlled by the motor drive based on the reference $Q_{m_{d}}$. $Q_{p}$ is the propeller load torque and $T_{p}$ the produced thrust. The friction torque is considered as a Coulomb plus a linear and nonlinear viscous effect. This model, motivated by experiments presented in [4] and [5], is written as

$$
Q_{f}(\omega)=k_{f_{1}} \frac{2}{\pi} \arctan \left(\frac{\omega}{\epsilon}\right)+k_{f_{2}} \omega+k_{f_{3}} \arctan \left(k_{f_{4}} \omega\right),
$$

where the coefficients $k_{f_{i}}$ and $\epsilon$ are constant and positive. In order to avoid the singularity in zero, the Coulomb effect, usually written as $\operatorname{aign}(\omega)$, has been replaced by the function $\frac{2}{\pi} \arctan \left(\frac{\omega}{\epsilon}\right)$ with a small positive $\epsilon$. The friction coefficients can be determined measuring the motor torque in steadystate conditions during test with the propeller in air. In this case the propeller load torque is zero and the motor torque corresponds to the friction torque. The propeller load torque $Q_{p}$ is represented by the nominal torque produced at zero advance speed (i.e. the propeller is at rest and not subjected to losses), plus a term $\Delta_{q}$ that incorporates torque losses:

$$
Q_{p}= \begin{cases}G_{Q_{p}}|\omega| \omega+\Delta_{q} & \omega \geq 0 \\ G_{Q_{n}}|\omega| \omega+\Delta_{q} & \omega<0 .\end{cases}
$$


The terms $G_{Q_{p}}$ and $G_{Q_{n}}$ are positive constants of different magnitudes since the propeller is usually not symmetric with respect to the shaft speed $\omega$. Neglecting the dynamics of the electrical part of the system (frequency converter, stator and rotor), which are usually much faster than the shaft dynamics, the control input is represented by $Q_{m}=Q_{m_{d}}$. Both $Q_{m}$ and $\omega$ are measured. The term $\Delta_{q}$ is represented by a Markov-like process with time constant $\tau$ driven by a bounded noise signal $w$ :

$$
\dot{\Delta}_{q}=-\frac{1}{\tau} \Delta_{q}+w .
$$

The noise $w$ represents the contribution of all the phenomena that can generate torque losses like variations in the vessel speed, propeller submergence, interaction between propeller and vessel hull, interaction between propellers and cross flows. Grouping the nonlinearities in the function $\psi(\omega)$, the system can be rewritten as:

$$
J_{m} \dot{\omega}=Q_{m}-\psi(\omega)-k_{f_{2}} \omega-\Delta_{q},
$$

where $\Delta_{q}$ is given by (4),

$\psi(\omega)=G_{Q_{p, n}}|\omega| \omega+k_{f_{1}} \frac{2}{\pi} \arctan \left(\frac{\omega}{\epsilon}\right)+k_{f_{3}} \arctan \left(k_{f_{4}} \omega\right)$,

and

$$
G_{Q_{p, n}}= \begin{cases}G_{Q_{p}}, & \omega \geq 0 \\ G_{Q_{n}}, & \omega<0\end{cases}
$$

\section{OBSERVER FOR TORQUE LOSS ESTIMATION}

A nonlinear observer with gain $l_{1}$ and $l_{2}$ is designed to estimate the torque loss $\hat{\Delta}_{q}$ and the shaft speed $\hat{\omega}=\hat{y}$ :

$$
\begin{aligned}
J_{m} \dot{\hat{\omega}} & =Q_{m}-\psi(\hat{\omega})-k_{f_{2}} \hat{\omega}-\hat{\Delta}_{q}+l_{1}(y-\hat{y}) \\
\dot{\hat{\Delta}}_{q} & =-\frac{1}{\tau} \hat{\Delta}_{q}+l_{2}(y-\hat{y}) \\
y & =\omega .
\end{aligned}
$$

Defining the observer error variables as $\tilde{\omega}=\omega-\hat{\omega}$ and $\tilde{\Delta}_{q}=\Delta_{q}-\hat{\Delta}_{q}$, and using the model in (4), (5), (6) and (7) and the observer in (8), (9) and (10), the observer error dynamics is written as

$$
\begin{aligned}
J_{m} \dot{\tilde{\omega}} & =-(\psi(\omega)-\psi(\hat{\omega}))-l_{1} \tilde{\omega}-k_{f_{2}} \tilde{\omega}-\tilde{\Delta}_{q} \\
\dot{\tilde{\Delta}}_{q} & =-\frac{1}{\tau} \tilde{\Delta}_{q}-l_{2} \tilde{\omega}+w .
\end{aligned}
$$

Proposition 1: If the gains $l_{1}$ and $l_{2}$ are chosen such that A1 $l_{1}>-k_{f_{2}}$,

$\mathbf{A 2}\left|\frac{a_{11}}{J_{m}}+p_{22} l_{2}\right|<2 \sqrt{a_{11} a_{22}\left(\frac{k_{f_{2}}+l_{1}}{\tau J_{m}}\right)}, a_{11}>0, a_{22}>0$,

then the error dynamics (11) and (12) is input-to-state stable (ISS) with respect to $w$.

Proof: Taking the Lyapunov function candidate $V_{o}\left(\tilde{\omega}, \tilde{\Delta}_{q}\right):=\frac{1}{2} a_{11} \tilde{\omega}^{2}+\frac{1}{2} a_{22} \tilde{\Delta}_{q}^{2}$ we can compute its time derivative along the trajectory of the observer error dynamics in (11) and (12):

$$
\begin{aligned}
\dot{V}_{o}= & -\frac{a_{11} k_{f_{2}}}{J_{m}} \tilde{\omega}^{2}-\frac{a_{11} l_{1}}{J_{m}} \tilde{\omega}^{2}-\frac{a_{22}}{\tau} \tilde{\Delta}_{q}^{2}-\frac{a_{11}}{J_{m}} \tilde{\omega} \tilde{\Delta}_{q} \\
& +a_{22} w \tilde{\Delta}_{q}-\frac{a_{11}}{J_{m}}[\psi(\omega)-\psi(\hat{\omega})] \tilde{\omega}-l_{2} a_{22} \tilde{\omega} \tilde{\Delta}_{q} .
\end{aligned}
$$

Noticing that the function $\psi(\cdot)$ belongs to the sector $[0, \infty]$ and is not decreasing, we have $\forall \omega, \hat{\omega}[\psi(\omega)-\psi(\hat{\omega})](\omega-\hat{\omega}) \geq 0$. Using this property, (13) becomes

$$
\begin{aligned}
\dot{V}_{o} & \leq-\frac{a_{11}\left(k_{f_{2}}+l_{1}\right)}{J_{m}} \tilde{\omega}^{2}-\frac{a_{22}}{\tau} \tilde{\Delta}_{q}^{2}-\left(\frac{a_{11}}{J_{m}}+a_{22} l_{2}\right) \tilde{\omega} \tilde{\Delta}_{q} \\
& +a_{22} \tilde{\Delta}_{q} w \\
& \leq-\tilde{e}_{o}^{T} Q_{1} \tilde{e}_{o}+a_{22} \tilde{\Delta}_{q} w
\end{aligned}
$$

where $\tilde{e}_{o}=\left[\begin{array}{ll}\tilde{\omega} & \tilde{\Delta}_{q}\end{array}\right]^{T}$ and

$$
Q_{1}=\left[\begin{array}{cc}
\frac{a_{11}\left(k_{f_{2}}+l_{1}\right)}{J_{m}} & \frac{1}{2}\left(\frac{a_{11}}{J_{m}}+a_{22} l_{2}\right) \\
\frac{1}{2}\left(\frac{a_{11}}{J_{m}}+a_{22} l_{2}\right) & \frac{a_{22}}{\tau}
\end{array}\right] .
$$

If the assumptions $\mathrm{A} 1$ and $\mathrm{A} 2$ hold, then $Q_{1}$ is positive definite and the origin of (11) and (12) with $w=0 \forall t$ is uniformly globally exponentially stable (UGES) since:

$$
\dot{V}_{o} \leq-\lambda_{\min }\left\{Q_{1}\right\}\left\|\tilde{e}_{o}\right\|^{2} .
$$

When $w \neq 0$ for some $t$, (14) can be written as follows:

$$
\begin{aligned}
\dot{V}_{o} & \leq-\lambda_{\min }\left\{Q_{1}\right\}\left\|\tilde{e}_{o}\right\|^{2}+a_{22}|w|\left\|\tilde{e}_{o}\right\| \\
& \leq-(1-\theta) \lambda_{\min }\left\{Q_{1}\right\}\left\|\tilde{e}_{o}\right\|^{2}-\theta \lambda_{\min }\left\{Q_{1}\right\}\left\|\tilde{e}_{o}\right\|^{2} \\
& +a_{22}|w|\left\|\tilde{e}_{o}\right\|,
\end{aligned}
$$

where $0<\theta<1$. For any $\left\|\tilde{e}_{o}\right\|$ such that

$$
\left\|\tilde{e}_{o}\right\| \geq \frac{a_{22}}{\theta \lambda_{\min }\left\{Q_{1}\right\}}|w|:=\rho_{1}(|w|),
$$

where $\rho_{1}(\cdot)$ is a (linear) class $\mathcal{K}$ function, we obtain:

$$
\dot{V}_{o} \leq-(1-\theta) \lambda_{\min }\left\{Q_{1}\right\}\left\|\tilde{e}_{o}\right\|^{2} \leq 0 .
$$

From Theorem 4.19 of [3], the system of (11) and (12) is ISS with respect to $w$.

The estimates $\hat{\omega}$ and $\hat{\Delta}_{q}$ can be used to compute an estimate of the propeller torque as:

$$
\hat{Q}_{p}=G_{Q_{p, n}}|\hat{\omega}| \hat{\omega}+\hat{\Delta}_{q} .
$$

\section{THRUST CONTROLLER}

We design a shaft speed controller which is derived as follows: first a desired torque $Q_{p_{d}}$ is computed from the desired thrust $T_{p_{d}}$ using the propeller characteristics and second, a desired shaft speed is computed from $Q_{p_{d}}$ and the estimated losses $\hat{\Delta}_{q}$.

The propeller characteristics is measured in steady-state conditions and is usually presented in the form of the nondimensional thrust and torque coefficients $K_{T}$ and $K_{Q}$. The coefficients are plotted as functions of the advance number $J$, given from:

$$
J=\frac{2 \pi u_{a}}{\omega D},
$$

where $D$ is the propeller disc diameter and $u_{a}$ is the advance speed (the speed of the inlet water to the propeller disc). The thrust and torque coefficients are computed from [10] as:

$$
K_{T}=\frac{4 \pi^{2} T_{p}}{\rho|\omega| \omega D^{4}}
$$




$$
K_{Q}=\frac{4 \pi^{2} Q_{p}}{\rho|\omega| \omega D^{5}} .
$$

The desired propeller torque is computed from (19) and (20) using the desired propeller thrust:

$$
Q_{p_{d}}=\frac{1}{\hat{G}_{Q T}(\hat{J})} T_{p_{d}}
$$

where $\hat{G}_{Q T}(\hat{J})$ is an estimate of

$$
G_{Q T}(J)=\frac{T_{p}}{Q_{p}}=\frac{\left.K_{T}\right|_{J}}{\left.D K_{Q}\right|_{J}},
$$

such that

$$
\hat{G}_{Q T}(\hat{J})=\frac{\left.K_{T}\right|_{\hat{J}}}{\left.D K_{Q}\right|_{\hat{J}}} .
$$

In order to compute $\hat{G}_{Q T}(\hat{J})$, an estimate $\hat{J}$ of the advance number is derived employing the estimated propeller torque. Using $\hat{Q}_{p}$ instead of $Q_{p}$ in (20) we compute the estimate $K_{Q}$. Combining the value of $\hat{K}_{Q}$ with the $K_{Q}$ curve of the propeller characteristics we can derive the value of $\hat{J}$. In Fig. 2 (a), it can be noticed that it is not possible to compute the exact value of $\hat{J}$ for values of $\hat{K}_{Q}$ in the area of the plot defined as zone 2 because the $K_{Q}$ curve in not invertible. In zone 1 and zone 3 the curve is invertible and $\hat{J}$ can be found accurately. In zone 2 we approximate the advance number with $\hat{J}=0$. This may be justified since in the zone 2 the ratio between thrust and torque does not change considerably. Since a propeller does not usually work for values of $J$ greater than 1, 1.2 and lower than -1.5 , the value of $\hat{J}$ has been limited to the range $[-1.5,1.12]$. Fig. 2 (b) shows $G_{Q T}(J)$ computed from the propeller characteristics and its approximation $\hat{G}_{Q T}(\hat{J})$. The plot refers to positive shaft speed $\omega$; the plot for negative speed is analogous.

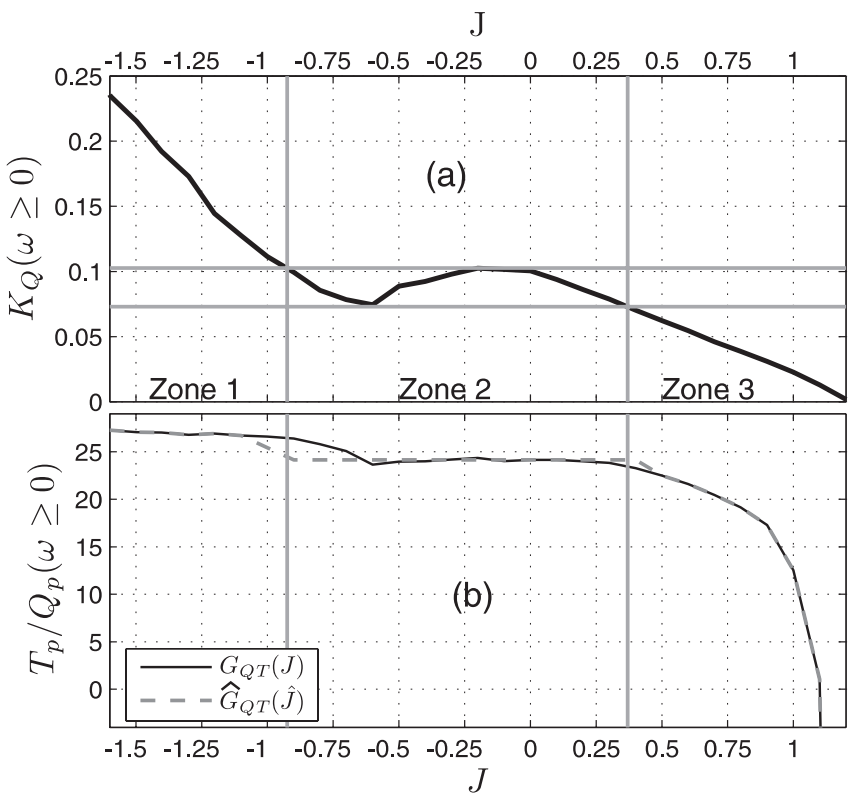

Fig. 2. $K_{Q}$ characteristic for $\omega \geq 0$ and the ratio between thrust and torque for $\omega \geq 0$.

In order to track the desired propeller torque $Q_{p_{d}}$, a shaft speed controller is designed. Given the desired propeller torque $Q_{p_{d}}$, the desired shaft speed $\bar{\omega}_{d}$ is computed by inverting (3) and using the estimated torque loss $\hat{\Delta}_{q}$ :

$$
\bar{\omega}_{d}=\sqrt{\frac{\left|Q_{p_{d}}-\hat{\Delta}_{q}\right|}{G_{Q_{p, n}}}} \operatorname{sign}\left(Q_{p_{d}}-\hat{\Delta}_{q}\right) .
$$

We design a controller to track the desired shaft speed $\bar{\omega}_{d}=f\left(Q_{p_{d}}, \omega, \hat{\Delta}_{q}\right)$. To generate a smooth reference signal $\omega_{d}$ and $\dot{\omega}_{d}$, we employ a second order low pass filter with cutoff frequency $\omega_{c}$ and relative damping factor $\xi$ :

$$
\ddot{\omega}_{d}+2 \omega_{c} \xi \dot{\omega}_{d}+\omega_{c}^{2} \omega_{d}=\omega_{c}^{2} \bar{\omega}_{d} .
$$

A block diagram of the overall system with the controller and the observer is shown in Fig. 3.

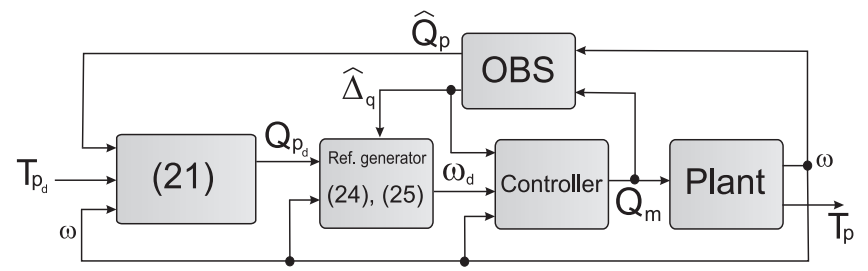

Fig. 3. Block diagram of the overall system.

We employ the following control law that includes a feedforward part, a proportional action and an integral action to ensure convergence in presence of constant disturbances:

$$
\begin{aligned}
Q_{m}= & J_{m} \dot{\omega}_{d}+\hat{\Delta}_{q}+\psi\left(-\gamma e_{1}+\omega_{d}\right)+k_{f_{2}} \omega_{d} \\
& -\left(k_{I}+\gamma k_{P}\right) e_{1}-k_{P} e_{2} .
\end{aligned}
$$

Defining the control errors $e_{1}=\int_{0}^{t}\left(\omega(\tau)-\omega_{d}(\tau)\right) d \tau$ and $e_{2}=\omega-\omega_{d}$, we can write the control error dynamics as:

$$
\begin{aligned}
\dot{e}_{1}= & e_{2} \\
\dot{e}_{2}= & -\frac{k_{f_{2}}}{J_{m}} e_{2}-\frac{1}{J_{m}}\left[\psi(\omega)-\psi\left(-\gamma e_{1}+\omega_{d}\right)\right]-\frac{k_{P}}{J_{m}} e_{2} \\
& -\frac{1}{J_{m}}\left(k_{I}+\gamma k_{P}\right) e_{1}-\frac{1}{J_{m}} \tilde{\Delta}_{q} .
\end{aligned}
$$
that

Proposition 2: If the gains $\gamma, k_{I}$ and $k_{P}$ are chosen such

B1 $\gamma>0$,

B2 $k_{P}>0, k_{P}>\gamma J_{m}-k_{f_{2}}$

B3 $k_{I}>0, k_{I}>-J_{m} \gamma^{2}+\gamma k_{f_{2}}$, $\tilde{\Delta}_{q}$

then the control error dynamics (27) is ISS with respect to

Proof: First we consider $\tilde{\Delta}_{q}=0$ and later we investigate its effect on (27). Let the Lyapunov function candidate be

$$
V=\frac{1}{2} e^{T} P e,
$$

where $e=\left[\begin{array}{ll}e_{1} & e_{2}\end{array}\right]^{T}$ and

$$
P=\left[\begin{array}{cc}
p_{11}+p_{22} \gamma^{2} & \gamma p_{22} \\
\gamma p_{22} & p_{22}
\end{array}\right],
$$


is positive definite. This implies $p_{11}>0$ and $p_{22}>0$. Taking its time derivative along the trajectory of the system (27) we obtain

$$
\begin{aligned}
\dot{V}= & -\frac{p_{22}}{J_{m}} \gamma\left(k_{I}+\gamma k_{P}\right) e_{1}^{2} \\
& +\left(p_{11}-p_{22}\left(-\gamma^{2}+\frac{k_{f_{2}}}{J_{m}} \gamma+2 \gamma \frac{k_{P}}{J_{m}}+\frac{k_{I}}{J_{m}}\right)\right) e_{1} e_{2} \\
& -p_{22}\left(-\gamma+\frac{k_{f_{2}}}{J_{m}}+\frac{k_{P}}{J_{m}}\right) e_{2}^{2} \\
& -p_{22} \frac{1}{J_{m}}\left[\psi(\omega)-\psi\left(-\gamma e_{1}+\omega_{d}\right)\right]\left(\gamma e_{1}+e_{2}\right),
\end{aligned}
$$

Since $\psi(\cdot)$ belongs to the sector $[0, \infty]$ and is not decreasing, the last term in (30) is always negative since

$$
\begin{aligned}
\forall \omega, e_{1}, \omega_{d}, \quad\left[\psi(\omega)-\psi\left(-\gamma e_{1}+\omega_{d}\right)\right]\left(\gamma e_{1}+e_{2}\right) \\
=[g(a)-g(b)](a-b) \geq 0,
\end{aligned}
$$

where $g=\psi, a=\omega, b=-\gamma e_{1}+\omega_{d}$. Choosing

$$
p_{11}=p_{22}\left(-\gamma^{2}+\frac{k_{f_{2}}}{J_{m}} \gamma+2 \gamma \frac{k_{P}}{J_{m}}+\frac{k_{I}}{J_{m}}\right),
$$

we cancel the cross term in (30). This is possible because, due to the assumptions B2 and B3 of proposition 2, the term inside the parenthesis in (32) is positive. Using (31) and (32), (30) becomes

$$
\begin{aligned}
\dot{V} & \leq-e^{T} Q_{2} e, \\
& \leq-\lambda_{\min }\left\{Q_{2}\right\}\|e\|^{2}
\end{aligned}
$$

where

$$
Q_{2}=\left[\begin{array}{cc}
\frac{p_{22}}{J_{m}} \gamma\left(k_{I}+\gamma k_{P}\right) & 0 \\
0 & p_{22}\left(-\gamma+\frac{k_{f_{2}}}{J_{m}}+\frac{k_{P}}{J_{m}}\right)
\end{array}\right]
$$

is positive definite if assumptions B1, B2 and B3 of proposition 2 are satisfied. The error dynamics (27) with $\tilde{\Delta}_{q}=0 \forall t$ is thus UGES. When $\tilde{\Delta}_{q} \neq 0$ for some $t$, (33) can be written as follows:

$$
\begin{aligned}
\dot{V} & \leq-\lambda_{\min }\left\{Q_{2}\right\}\|e\|^{2}-\frac{p_{22}}{J_{m}} \tilde{\Delta}_{q}\left(\gamma e_{1}+e_{2}\right) \\
& \leq-(1-\theta) \lambda_{\min }\left\{Q_{2}\right\}\|e\|^{2}-\theta \lambda_{\min }\left\{Q_{1}\right\}\|e\|^{2} \\
& +\frac{p_{22}}{J_{m}}\left|\tilde{\Delta}_{q}\right| \sqrt{1+\gamma^{2}}\|e\|,
\end{aligned}
$$

where $0<\theta<1$. For any $\|e\|$ such that

$$
\|e\| \geq \frac{p_{22} \sqrt{1+\gamma^{2}}}{\theta J_{m} \lambda_{\min }\left\{Q_{2}\right\}}\left|\tilde{\Delta}_{q}\right|:=\rho_{2}\left(\left|\tilde{\Delta}_{q}\right|\right),
$$

where $\rho_{2}(\cdot)$ is a (linear) class $\mathcal{K}$ function, we obtain:

$$
\dot{V} \leq-(1-\theta) \lambda_{\min }\left\{Q_{2}\right\}\|e\|^{2} \leq 0 .
$$

From Theorem 4.19 of [3], the system of (27) is ISS with respect to $\tilde{\Delta}_{q}$ and the control error is uniformly ultimately bounded by $\rho_{2}\left(\sup _{t>t_{0}}\left(\left|\tilde{\Delta}_{q}\right|\right)\right)$.

Furthermore the bound of the control error decreases when increasing the control gains, since it results in an increase of the value of $\lambda_{\min }\left\{Q_{2}\right\}$. The observer/controller error dynamics can be considered as a cascaded system where the observer error $\tilde{\Delta}_{q}$ drives the control error dynamics. The observer error dynamics $\tilde{\Delta}_{q}$ is bounded and ISS with respect to $w$, implying that the control error dynamics $e$ is bounded. Due to the boundness of $T_{p_{d}}$ and from the relation (21) between thrust and torque, also $Q_{p_{d}}$ is bounded. The torque loss estimation $\hat{\Delta}_{q}$ is also bounded due to the property of the observer, therefore the desired shaft speed $\bar{\omega}_{d}$ and thus $\omega_{d}$ are bounded. This last condition implies boundness of the shaft speed $\omega$ since $e$ is confined. The propeller thrust is thus bounded and converges to a ball around the desired thrust.

\section{EXPERIMENTAL RESULTS}

\section{A. Setup}

The tests were performed at the MCLab ( http://www.itk.ntnu.no/marinkyb/MCLab/ ), an experimental laboratory equipped with a towing carriage that can reach a maximum speed of $2 \mathrm{~m} / \mathrm{s}$. The rig with motor, underwater housing, shaft and propeller was attached to the towing carriage in order to move it through the water. The propeller was a fixed pitch propeller with diameter $25 \mathrm{~cm}$. The

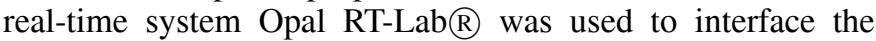

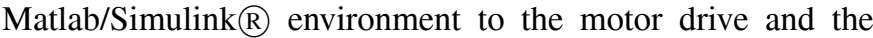
sensors. We employed a three phase brushless motor and a drive equipped with a built-in torque controller sufficiently fast to consider its dynamics neglectable. The thrust was measured with an inductive transducer and the torque was measured with a strain gauge transducer placed on the propeller shaft. A sketch of the setup is shown in Fig. 4. Fig. 7 shows a picture of the propeller system.

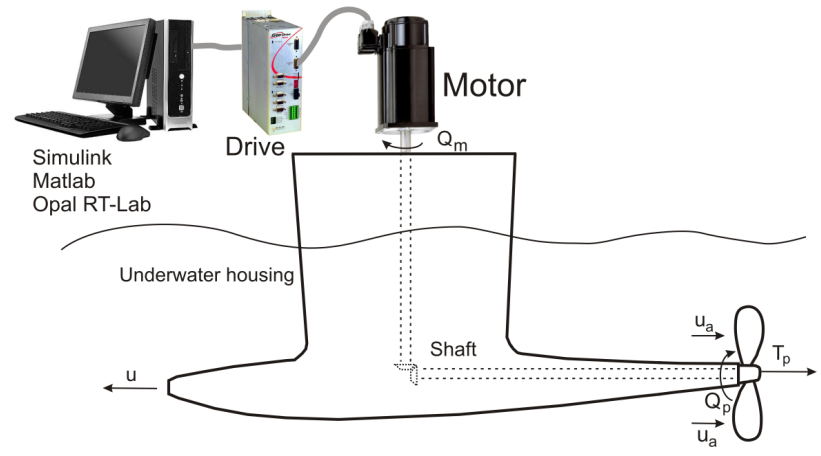

Fig. 4. Sketch of the experimental setup.

\section{B. Results}

The thrust controller scheme has been validated with the parameters reported in Table I.

An experiment has been carried out to test the controller in the four-quadrants of the plane composed by the advance speed and the shaft speed. The desired thrust was a $80 \mathrm{~N}$ sinusoidal wave with period $25 \mathrm{~s}$ while the advance speed $u_{a}$ was varied from zero to $0.5 \mathrm{~m} / \mathrm{s}$ and to $-0.5 \mathrm{~m} / \mathrm{s}$. Fig. 5 (a) shows that the measured thrust, plotted after filtering, tracks the demanded thrust satisfactorily. The tracking error, shown in Fig. 5 (b), presents spikes when the shaft speed crosses zero. This may be due to the use of a static friction torque model while the real one might present a dynamic behaviour. The use of a dynamic model for the friction torque may therefore improve the performance of the controller at values of $\omega$ close to zero. Fig. 5 (c) shows the desired shaft speed $\omega_{d}$ and the filtered shaft speed $\omega$ for the same experiment. It is possible to see that the measurement follows precisely the desired one. 
Fig. 5 (d) shows the control input. The desired motor torque $Q_{m_{d}}$ and the measured $Q_{m}$ are almost indistinguishable. This is due to the high precision built-in torque controller of the motor drive. This also confirms that the dynamics of the motor is negligible with respect the dynamics of the propeller shaft.

Some tests where performed to compare the thrust controller scheme developed in this paper with the traditional torque and shaft speed controllers. Experimental validation of torque and shaft speed control has been presented in [9] and [8]. Since the value of the advance speed is usually unknown, both the traditional torque and shaft speed controller uses the nominal values of the propeller characteristics for $J=0$. The propeller working condition is not accounted for in the traditional controller schemes. For a torque controller the demanded motor torque is computed as [8]:

$$
Q_{m_{d}}=\frac{\left.D K_{Q}\right|_{J=0}}{\left.K_{T}\right|_{J=0}} T_{p_{d}} .
$$

For the shaft speed controller the demanded shaft speed $\omega_{d}$ is calculated as [8]:

$$
\omega_{d}=\sqrt{\frac{4 \pi^{2}\left|T_{p_{d}}\right|}{\left.\rho K_{T}\right|_{J=0} D^{4}}} \operatorname{sign}\left(T_{p_{d}}\right) .
$$

Fig. 6 (a) shows the propeller thrust measured during experiments employing different controllers. The demanded thrust was kept constant at $50 \mathrm{~N}$ while varying the advance speed. The controller developed in this paper provides the best results because it is able to compensate for losses due to the change of the advance speed. When the advance speed is zero all the controllers provide the demanded thrust. When the advance speed increases, the thrust controller increases the shaft speed in order to keep the thrust constant, as shown in Fig. 6 (b). The traditional shaft speed controller gives the worst results. The torque controller increases the shaft speed but not sufficiently to compensate for the losses.

When the advance speed becomes negative, the propeller tries to reverse the inlet flow and a recirculation zone (often called a ring vortex) occurs [11]. This is due to the interaction between the inlet flow and the reversed flow. The flow becomes unsteady and this can cause oscillations in the propeller thrust. Also in this condition the thrust controller provides the best results. The thrust is oscillating, but the average is around the demanded thrust. The thrust controller gives more rapid variations in shaft speed than the other controllers. This may increase wear-and-tear of the mechanical parts of the thruster. This problem will be addressed in a future work. As for positive advance speed, the traditional shaft speed controller gives the worst result in terms of produced thrust, while the torque controller produces a less oscillatory thrust with average below the requested one.

\section{CONCLUSION}

In this paper a nonlinear thrust controller for a marine propeller designed to work in four-quadrant operations has been presented. It was a shaft speed controller where the desired velocity was computed based on the desired propeller thrust and on the torque losses, estimated with a nonlinear observer. Experimental results showed the effectiveness of the controller, which was compared with the traditional shaft
TABLE I

OBSERVER AND CONTROLLER PARAMETERS

\begin{tabular}{ll|ll}
\hline Parameter & Value & Parameter & Value \\
\hline$J_{m}\left[\mathrm{~kg} \cdot \mathrm{m}^{2}\right]$ & $5.84 \cdot 10^{-3}$ & $l_{1}$ & 3 \\
$k_{f 1}\left[\frac{\mathrm{s} \cdot \mathrm{N}}{\mathrm{m} \cdot \mathrm{rad}}\right]$ & $11.3 \cdot 10^{-2}$ & $l_{2}$ & -160 \\
$k_{f_{2}\left[\frac{\mathrm{s} \cdot \mathrm{N}}{\mathrm{m} \cdot \mathrm{rad}}\right]}$ & $1.29 \cdot 10^{-2}$ & $k_{p}$ & 0.09 \\
$k_{f_{3}}\left[\frac{\mathrm{s} \cdot \mathrm{N}}{\mathrm{m} \cdot \mathrm{rad}}\right]$ & $6.96 \cdot 10^{-1}$ & $k_{I}$ & 0.2 \\
$k_{f_{4}}$ & $8.03 \cdot 10^{-1}$ & $\gamma$ & 1.1 \\
$\epsilon$ & $1 \cdot 10^{-6}$ & $\omega_{c}[\mathrm{rad} / \mathrm{s}]$ & 31.42 \\
$\tau[s]$ & 10 & $\xi$ & 1 \\
\hline
\end{tabular}

speed and torque controllers, showing improved performance with respect to the thrust production.

\section{ACKNOWLEDGMENT}

The authors acknowledge Johannes Tjønnås for valuable suggestions and discussions. The Research Council of Norway is acknowledged as the main sponsor of this project.

\section{REFERENCES}

[1] T. I. Fossen and M. Blanke, "Nonlinear output feedback control of underwater vehicle propellers using feedback from estimated axial flow velocity," IEEE Journal of Oceanic Eng., vol. 25, no. 2, April 2000.

[2] C. Guibert, E. Foulon, N. Aït-Ahmed, and L. Loron, "Thrust control of electric marine thrusters," Industrial Electronics Society. IECON 2005. 32nd Annual Conference of IEEE, 2005.

[3] H. K. Khalil, Nonlinear Systems, 3rd ed. Prentice Hall, 2000.

[4] L. Pivano, T. I. Fossen, and T. A. Johansen, "Nonlinear model identification of a marine propeller over four-quadrant operations," 14th IFAC Symposium on System Identification, SYSID, Newcastle, Australia, 2006.

[5] L. Pivano, Ø. N. Smogeli, T. A. Johansen, and T. I. Fossen, "Experimental Validation of a Marine Propeller Thrust Estimation Scheme," 7th IFAC Conference on Manoeuvring and Control of Marine Craft, Lisbon, 2006.

[6] Ø. N. Smogeli, "Control of Marine Propellers: From Normal to Extreme Conditions," Ph.D. dissertation, Department of Marine Technology, Norwegian University of Science and Technology (NTNU), Trondheim, Norway, September 2006.

[7] Ø. N. Smogeli, J. Hansen, A. J. Sørensen, and T. A. Johansen, “Anti-spin control for marine propulsion systems," Proc. 43rd IEEE Conference on Decision and Control. Paradise Island, Bahamas, 2004.

[8] Ø. N. Smogeli, E. Ruth, and A. J. Sorensen, "Experimental validation of power and torque thruster control," Proceedings of the 2005 IEEE International Symposium on, Mediterrean Conference on Control and Automation, pp. 1506- 1511, 2005.

[9] A. J. Sørensen and $\varnothing$. N. Smogeli, "Torque and power control of electrically driven propellers on ships," Accepted for publication in the IEEE Journal of Oceanic Engineering, 2006.

[10] W. P. A. Van Lammeren, J. D. V. Manen, and M. W. C. Oosterveld, "The Wageningen B-Screw Series," Transactions of SNAME, 1969.

[11] M. Vysohlid and K. Mahesh, "Large-Eddy Simulation of Propeller Crashback," APS Meeting Abstracts, Nov. 2004.

[12] L. L. Whitcomb and D. Yoerger, "Developement, comparison, and preliminary experimental validation of nonlinear dynamic thruster models," IEEE Journal of Oceanic Engineering, vol. 24, no. 4, pp. 481-494, Oct. 1999. 

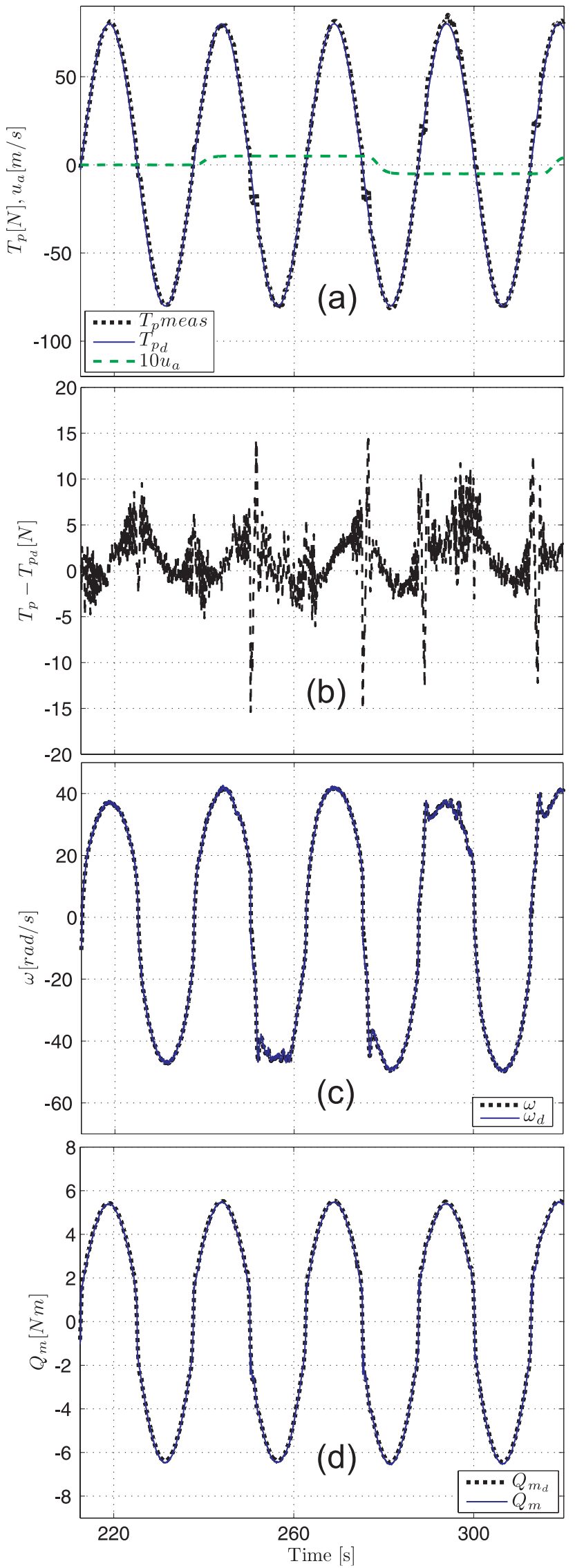

Fig. 5. Experiment with a sinusoidal demanded thrust: propeller thrust and advance speed (a), thrust tracking error (b), shaft speed (c) and motor torque (d).
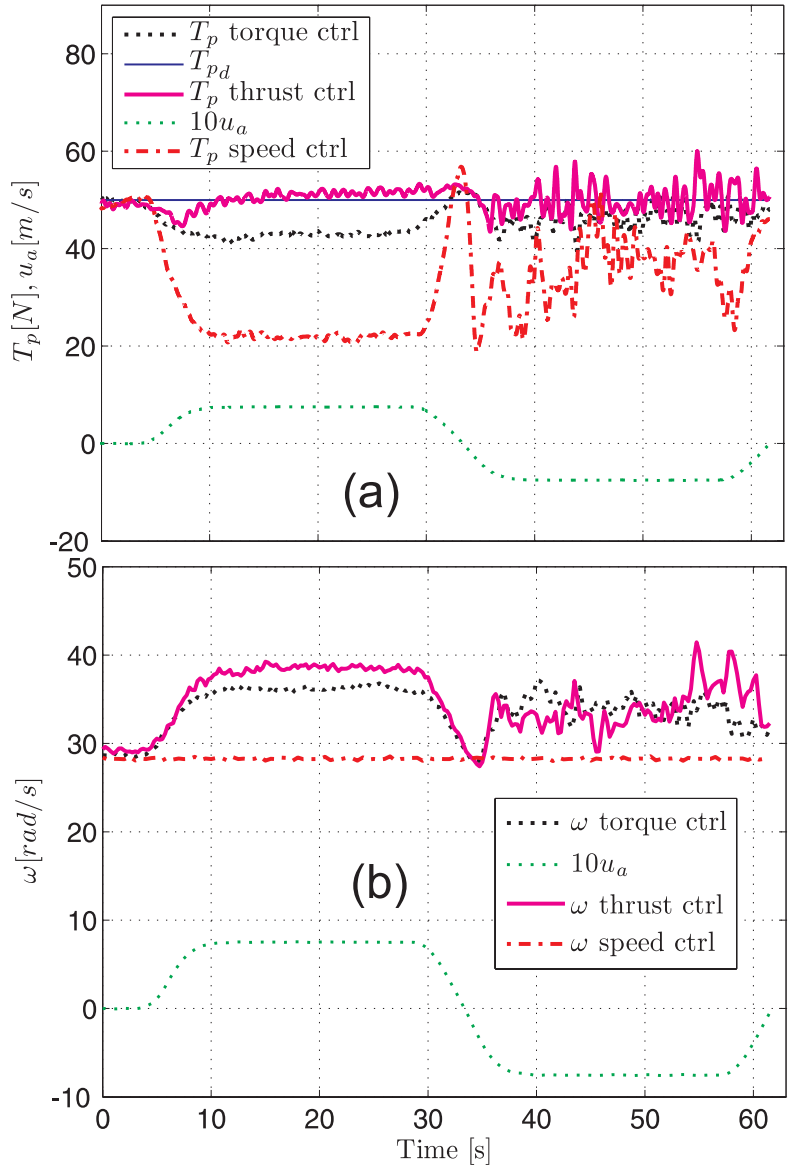

Fig. 6. Comparison among different controllers: propeller thrust (a) and shaft speed (b).

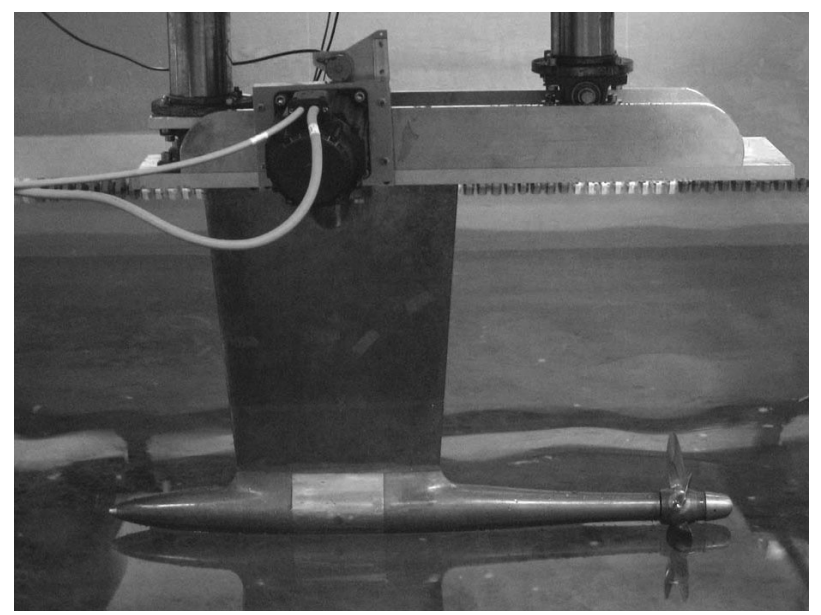

Fig. 7. Picture of the propeller system. 